\title{
The role of integration in recognition failure and action memory
}

\author{
REZA KORMI-NOURI and LARS-GÖRAN NILSSON \\ Stockholm University, Stockholm, Sweden
}

\begin{abstract}
In three experiments, we studied memory for action events with respect to exceptions from the Tulving-Wiseman function demonstrated in experiments on recognition failure of recallable words. In Experiment 1, we examined exceptions of poor integration in a regular recognition failure condition (i.e., recognition of targets without contextual cues, followed by recall of targets in the presence of contextual cues). In Experiment 2, we examined exceptions of cue overlap in which subjects also had access to the information of contextual cues at recognition test. In Experiment 3, we attempted to equate the levels of recognition across the action and verbal encoding. In addition, the cue overlap and no-cue overlap conditions were studied in a within-subjects design. Results from the three experiments indicated that encoding enactment (episodic integration) and conceptual integration (semantic integration) are related to each other. As a consequence of this relationship, there is a larger independence between recognition and recall of well-integrated items with encoding enactment. On the other hand, for the poorly integrated items without encoding enactment, there is a larger dependence between recognition and recall. Even in the cue overlap condition, where there is a case of large dependence between recognition and recall, the same pattern of data was observed. The results are discussed in terms of an episodic integration view of encoding enactment.
\end{abstract}

Verbal materials have been used as the to-be-remembered (TBR) information in memory experiments for more than 100 years. About 15 years ago, a new experimental paradigm for studying memory functions was developed ( $\mathrm{Co}-$ hen, 1981; Engelkamp \& Krumnacker, 1980; Saltz \& Donnenwerth-Nolan, 1981). This paradigm is usually referred to as an action memory or a subject-performed task (SPT), which is compared with a traditional verbal task (VT) for assessing memory performance. The main difference between SPTs and VTs consists in enactment at encoding. In SPT encoding, subjects are required to perform a number of minitasks (e.g., roll the ball, break the match). In the control condition (i.e., VT encoding), subjects receive the verbal commands without performing them. The typical finding in SPT experiments is that recall and recognition of enacted commands is superior to recall and recognition of commands without any enactment (see Cohen, 1989, for a review).

Several theories have been proposed to account for the superiority of SPT recall over VT recall. Cohen (1981, 1983; Cohen \& Stewart, 1982) proposed a nonstrategic view of encoding of SPTs. VTs can benefit from rehearsal,

This research was supported by a grant to L.-G.N. from the Swedish Council for Research in the Humanities and Social Science. We would like to thank Michael Masson, Elliot Hirshman, Vincenza Gruppuso, Art Flexser, and one anonymous reviewer for their valuable comments on an earlier draft of the manuscript. Correspondence concerning this article should be sent to R. Kormi-Nouri or L.-G. Nilsson, Department of Psychology, Stockholm University, S-106 91 Stockholm, Sweden (e-mail: lnn@psychology.su.se).

-Accepted by previous editor, Geoffrey R. Loftus or from organizational and associational strategies during presentation of items, whereas such encoding strategies appear to be of little importance in SPTs. Cohen (1981, 1983; Cohen \& Stewart, 1982) concluded that SPT learning provides an optimal form of encoding and thereby produces a better memory performance than does VT encoding.

Bäckman and Nilsson $(1984,1985)$ proposed that the multimodal and rich encoding for SPTs causes the superior memory performance for SPTs. SPTs are multimodal in the sense that several systems (auditory, visual, tactual, and in some cases, gustatory and olfactory) are involved during encoding, whereas VTs are usually presented unimodally (visual or auditory) and in some cases bimodally (visual and auditory). SPT learning leads automatically to a rich multimodal encoding of the event. In addition, Bäckman and Nilsson assumed that SPTs are nonstrategic in some but not all respects. They proposed a dual conception of SPTs, verbal and physical (Bäckman, Nilsson, \& Chalom, 1986), and they assumed that the encoding of the verbal component of SPTs is strategic, whereas the encoding of the physical component (weight, color) of SPTs is nonstrategic (Bäckman, Nilsson, Herlitz, Nyberg, \& Stigsdotter, 1991; Bäckman, Nilsson, \& Kormi-Nouri,1993).

Engelkamp and Zimmer (1983, 1984) proposed a motor program in addition to the use of verbal and visual programs. They assumed that motor, verbal, and visual encodings are independent of each other in the sense that they have different representations, codes, and modalityspecific properties. Motor encoding is of special importance and more efficient than verbal and visual encoding, thereby producing the SPT effect. 
Kormi-Nouri (1994) proposed that SPT memory is a type of verbal encoding and that its nature is both strategic and integrative. Kormi-Nouri, Nyberg, and Nilsson (1994) demonstrated that SPTs were recalled better by verbal cues than by motor cues, indicating that SPTs are not encoded in motor codes. The level of integration (conceptual linkage between verb and noun) which was manipulated within the items did not change the pattern of results. In the Kormi-Nouri, Nilsson, and Bäckman (1994) study, it was found that both verbal and physical features of SPTs are equally attention demanding. Furthermore, Kormi-Nouri (1995) demonstrated that there was no substantial difference between SPT memory and VT memory with respect to the use of strategies at encoding: Both SPT and VT memory were affected by the semantic relation between verb and noun. The level of semantic integration for each individual item was manipulated by the conceptual linkage between verb and noun: A high semantic integration was called a well-integrated item (WI; e.g., read the book, write with the pen), whereas a low semantic integration was called a poorly integrated item (PI; e.g., lift the paper, scratch with the button). The main difference between SPT and VT memory was found to be due to integration produced by encoding enactment. That is, enactment produces a high integration between action verb and noun (object) for each particular item at the time of encoding. This was proposed as episodic integration. As a consequence of this, the two components of an action event are encoded as a single memory unit or as strongly connected memory units. On the other hand, nonenactment (verbal encoding) produces a lower form of integration. That is, verbs and nouns are encoded separately or are loosely connected.

The present study is based on the episodic integration view. It was hypothesized that if these two types of items (WIs and SPTs) are similar with respect to the integration notion, semantically or episodically, they should be correlated with each other. That is, combining semantic and episodic integration within a single condition should strengthen the effect that each has on recognition and recall (i.e., enactment should strengthen the extent to which the item is semantically integrated, and semantic integration should strengthen the extent to which the item is episodically integrated). Given that the enactment encoding of WIs increases semantic and episodic integration between action verb and object, then (1) WI items should be recalled and recognized better by SPT encoding than by VT encoding, and (2) the effect of SPT encoding should be higher for WI items than for PI items.

Our main concern in the present study was to examine the idea of interrelation between semantic and episodic integration, high episodic integration and high semantic integration (i.e., memory for SPT/WIs) versus low episodic integration and low semantic integration (i.e., memory for VT/PIs) in the recognition failure paradigm, which represents an empirical relationship between recognition and recall (Nilsson, Law, \& Tulving, 1988). This para- digm is known as recognition failure for recallable words (Tulving \& Thomson, 1973).

In recognition failure experiments, a TBR item is encoded with a contextual item at the study phase. At test, the TBR item is first recognized in the absence of the context. Second, following the recognition test, the TBR is recalled in the presence of the context. In these situations, subjects frequently recall the item that they failed to recognize in the preceding recognition test. According to the Tulving and Wiseman (1975) function, ${ }^{1}$ the relation between these two tests is invariant across a large number of variables, showing a moderate dependency (see Nilsson \& Gardiner, 1993, for a review). Yet despite the remarkable invariance that has been observed in recognition failure experiments in general, a few exceptions to this regularity have also been observed. One type of exception to the Tulving-Wiseman function occurs because of poor integration at the time of encoding (Tulving, 1983). Another type of exception is cue overlap, which can be due to knowledge of the contextual cue at recognition. As was discussed by Nilsson et al. (1988), a poor integration of two parts of an item and a high degree of cue overlap increase the degree of dependency between recognition and recall.

The crucial role of integration of context cue and target item in the recognition failure paradigm and the empirical regularities demonstrated (Nilsson \& Gardiner, 1993) make this paradigm interesting for testing the integration theory proposed by Kormi-Nouri (1995). More specifically, the integration variable was studied with the purpose for exploring whether there is a relationship between episodic integration, which has been used in action memory experiments, and conceptual integration, which has been used in recognition failure experiments. To our knowledge, there is only one study (Svensson \& Nilsson, 1989 ) in which enactment encoding has been examined in the context of the recognition failure paradigm. Svensson and Nilsson found that SPT, as a case of highly integrated items, increases the degree of independence between recognition and recall. VT, on the other hand, as a case of poorly integrated items, increases the degree of dependence between recognition and recall. In line with this previous study, it was expected that there is a higher degree of independence between recognition and recall for SPTs than for VTs. This was expected because SPTs produce a higher degree of episodic integration between the two components of an action, whereas VTs produce a lower degree of episodic integration. In addition, it was expected that the combination of semantic and episodic integration (i.e., encoding of SPT/WI items) should create the highest level of integration and consequently should result in the most profound independence between recognition and recall. On the other hand, the lowest degree of integration (i.e., encoding of VT/PI items) should result in a strong dependence between recognition and recall.

In regard to the exception of cue overlap, Svensson and Nilsson (1989) found a somewhat different pattern 
of recognition failure data for SPTs and VTs than they did for the exception of poor integration. Both SPTs and VTs exhibited an increased dependence between recognition and cued recall. In the present study, using another experimental manipulation of cue overlap, we predicted that the highly integrated condition of SPT/WIs should override the influence of dependence (between recall and recognition) produced by cue overlap, thus still producing independence (i.e., a lower level of dependence) between recognition and recall. For VT/PIs, on the other hand, there is no such strong integration which can compensate for the dependence produced by cue overlap, and there should still be dependence between recognition and recall.

The present study comprised three experiments. In Experiment 1, we studied the notion of high integration (SPT/WI) and of poor integration (VT/PI) in a regular recognition failure experiment (i.e., a recognition test, including only targets, was followed by a cued recall test). In Experiment 2, recognition and cued recall of SPT/WIs and VT/PIs were examined in a cue overlap condition: Recognition of whole items (including both contextual cues and targets) was followed by cued recall of items. In Experiment 3, the no-cue overlap and cue overlap condition were examined in a within-subjects design where the levels of recognition for SPTs and VTs were almost equalized.

\section{EXPERIMENT 1}

The main purpose of Experiment 1 was to examine the effects of semantic and episodic integration in the recognition failure paradigm (Tulving \& Thomson, 1973). In line with the previous study (Kormi-Nouri, 1995), it was expected that the combination of low semantic and low episodic integration (VT/PI) produces the lowest integration between verb and noun of an item, thereby producing the highest degree of dependence between recognition and recall. On the basis of the same type of reasoning, a high semantic and a high episodic integration (SPT/WI) should result in a higher level of integration; hence, one should expect a higher degree of independence between recognition and recall. Although the direction of each of these predictions is clear, it was not transparent whether both the VT/PI and SPT/WI conditions would deviate significantly from the Tulving-Wiseman function, since this function itself expresses a certain degree of dependence.

\section{Method}

Design. The design of Experiment 1 was 2 (type of integration: WI vs. PI) $\times 2$ (type of encoding: SPT vs. VT) $\times 2$ (type of test: recognition vs. recall) $\times 2$ (type of cue: verb vs. noun). The first three factors were varied within subjects, whereas the last factor was manipulated between subjects.

Subjects. Twenty-four students were randomly assigned to two conditions: verb cue and noun cue at test. Their age ranges were 19-32 $(M=23.00)$ for the verb cue condition and 19-28 $(M=22.33)$ for the noun cue condition. The subjects were paid for their participation. The experiment lasted approximately $30 \mathrm{~min}$.

Materials. A list of 48 commands with two presentation orders comprised the TBR items. The items were selected from the pool of items in the normative study (Kormi-Nouri, 1995). Half of the items were selected as WIs (e.g., write with the pen, cut with the knife, read the book), and the other half, as PIs (e.g., lift the paper, point at the wallet, look at the stone). For each type of item, half of the items were encoded as SPTs and the other half as VTs. The assignment of items was counterbalanced to the SPT or VT encoding over the subjects. All items appeared as SPTs and VTs taken across all subjects. Not more than two WIs or PIs, and SPTs or VTs were allowed to appear in succession.

Procedure. Each subject was tested individually. The items were shown on cards by the experimenter one by one, at a rate of $6 \mathrm{sec}$ per item. The interstimulus interval was $3 \mathrm{sec}$. All subjects were instructed to remember the whole item for a subsequent memory test. For VTs, the subjects were instructed to read the item only, whereas for SPTs, the subjects were instructed to read and perform the action indicated by the item. Real objects were used in SPTs: The objects were given to the subjects at the time of presentation of items and were then hidden after they performed the items. The experimenter said "sentence" before presenting VTs and "action" before presenting SPTs. All subjects were given one example of a VT and one of an SPT before the study list was presented.

After the presentation of the last item in the study list, the subjects were given a 30 -item vocabulary test. The main reason for administrating the vocabulary test was to eliminate any effects of shortterm memory. However, another reason was to assess word comprehension for the subject. The two groups of subjects were not different with respect to the vocabulary test $(p>.40)$.

Following the vocabulary test, each subject was given two tests: first, a recognition test, and second, a recall test. At recognition, half of the subjects were asked to recognize the noun targets (verbs as contextual cues were not presented) among noun distractors, whereas the other half were asked to recognize the verb targets (nouns as contextual cues were not presented) among verb distractors. There were $\mathbf{4 8}$ targets and $\mathbf{4 8}$ distractors presented in a random order for a free-choice recognition test. The target and distractor items were written one by one on the test sheet for a yes/no decision regarding whether the items had been presented in the study list. In order to avoid ceiling effects in the recognition test, the distractors were selected so that they would be similar to the targets with respect to semantic category of nouns (e.g., book, newspaper; bag, sack) and verbs (e.g., write, draw; talk, speak). The recall test was given to the subjects corresponding to the type of recognition test (i.e., recognition-noun target/recall-verb cue; recognition-verb target/recall-noun cue). For the sake of simplicity, copy cue stands for the targets at recognition test, and contextual cue stands for the cues at recall test given to subjects to recall the target items.

\section{Results and Discussion}

A strict scoring of recall was used. That is, responses were accepted only if they were exactly the same as in the study list. The results are shown in Table 1.

Overall analysis for recognition and recall. A 2 (list) $\times 2$ (type of cue: verb/noun) $\times 2$ (type of integration:

Table 1

Mean Recognition (Rn) and Cued Recall (Rc) Probabilities and Observed and Predicted Conditional Recognition Probabilities for Experiment 1

\begin{tabular}{|c|c|c|c|c|c|c|c|}
\hline \multirow[b]{2}{*}{ Condition } & \multicolumn{2}{|c|}{$\mathrm{Rn}$} & \multicolumn{2}{|c|}{$\mathrm{Rc}$} & \multicolumn{2}{|c|}{$p(\mathrm{Rn} \mid \mathrm{Rc})$} & \multirow[b]{2}{*}{$N$} \\
\hline & $M$ & $S D$ & $M$ & $S D$ & Observed & Predicted & \\
\hline SPT/WI & .92 & .07 & .86 & .14 & $.93^{*}$ & .96 & 288 \\
\hline SPT/PI & .73 & .19 & .44 & .20 & .84 & .83 & 288 \\
\hline VT/WI & .58 & .23 & .78 & .19 & .70 & .71 & 288 \\
\hline $\mathrm{VT} / \mathrm{PI}$ & .53 & .22 & .15 & .19 & .67 & .65 & 288 \\
\hline
\end{tabular}

${ }^{*} p<.05$ (two tailed binomial test). 
WI/PI) $\times 2$ (type of encoding: SPT/VT) $\times 2$ (type of test: recognition/recall) (ANOVA) was conducted. There was no difference between the two lists $(p>90)$. Thus, the data were collapsed across this factor. The ANOVA showed significant main effects of type of integration $\left[F(1,22)=166.50, M S_{\mathrm{e}}=4.61, p<.001\right]$, type of encoding $\left[F(1,22)=66.64, M S_{\mathrm{e}}=6.04, p<.001\right]$, and type of test $\left[F(1,22)=30.95, M S_{\mathrm{e}}=3.39, p<.001\right]$. The interaction between type of integration and type of test was significant $\left[F(1,22)=95.69, M S_{\mathrm{e}}=3.48, p<.001\right]$, indicating that WIs produced a better result in recall than in recognition, whereas PIs were higher in recognition than in recall. The interaction between type of encoding and type of test was significant $\left[F(1,22)=5.29, M S_{\mathrm{e}}=\right.$ $1.74, p<.05$ ], indicating that the SPT-VT difference (SPT effect) was higher in recognition than in recall. In addition, the three-way interaction of type of integration, type of encoding, and type of test was significant $[F(1,22)=$ $\left.35.29, M S_{\mathrm{e}}=1.14, p<.001\right]$. For WIs, the SPT effect was higher in recognition than in recall, whereas for PI, it was higher in recall than in recognition.

With regard to type of cue (noun or verb), in general, there was no effect $(p>.80)$. Moreover, there was no overall interaction between type of cue and type of test $(p>.20)$. However, some other interactions were significant: the three-way interaction of type of cue, type of integration, and type of test $\left[F(1,22)=5.98, M S_{\mathrm{e}}=3.35\right.$, $p<.05]$; the three-way interaction of type of cue, type of encoding, and type of test $\left[F(1,22)=5.80, M S_{\mathrm{e}}=1.74\right.$, $p<.05]$; and, finally, the four-way interaction of type of cue, type of integration, type of encoding, and type of test $\left[F(1,22)=8.55, M S_{\mathrm{e}}=43.53, p<.01\right]$. The most interesting implication of these interactions is that no interaction included SPTs with respect to type of cue, whereas there was an interaction for VTs: VT/PI items were recognized better in the verb cue condition (noun targets) than in the noun cue condition (verb targets), whereas they were recalled better with the noun cues than with the verb cues.

In line with the predictions, the results showed that SPT memory, like VT memory, was affected by the semantic integration in both recognition and recall, and that the enactment effect was seen in both recognition and recall. The latter finding replicated the results of studies by Svensson and Nilsson (1989), and Nilsson and Craik (1990). However, unlike in these studies, and in line with Mohr, Engelkamp, and Zimmer (1989), the overall results of the present experiment showed that the effect of enactment was more pronounced for recognition than for recall. It should be noted that this effect was observed only for the noun cue condition, whereas in the verb cue condition, there was no difference for the SPT effect in recognition and recall. The possible explanation is the type of item. This will be discussed next.

In line with the study by Shaps and Nilsson (1980), memory performance for WIs was better in recall than in recognition, whereas memory performance for PIs was better in recognition than in recall. In addition, in line with the previous study (Kormi-Nouri, 1995), there was a differential effect of enactment for WIs and PIs. The ef- fect of encoding enactment was most pronounced either in recognition of WIs or in recall of PIs. These findings support the idea that the effect of enactment can be seen with respect to task effortfulness (Helstrup, 1986, 1987). This means that enactment serves as encoding support and its effect emerges whenever the memory task invites one to employ learning efforts. For WIs, recall is less effortful than recognition, whereas for PIs, recall is more effortful than recognition. Thus, on the basis of the assumption of similar processing for encoding and retrieval, it might be concluded that SPT encoding, in general, increases the episodic integration between verb and noun, and that its efficiency depends on the difficulty of the task.

In SPT encoding, the finding of better recognition for well-integrated items than for poorly integrated items is inconsistent with the line of reasoning of Mohr et al. (1989). They found that the SPT effect was more pronounced in recognition than in free recall and that the SPT effect was reduced for unusual (bizzare) items (e.g., bury the revolver, plant the hammer) (as an index of increasing item-specific information for VTs) in comparison with usual items (e.g., read the newspaper, water the flower). Thus, according to Mohr et al., whenever the degree of item-specific information is increased for verbal tasks, the effect of enactment is reduced (this should be the case in recognition more than in recall, since recognition is based on item-specific information more than recall is). We agree with these authors that SPT encoding improves itemspecific information. However, we disagree with these authors that the effect of enactment should usually be better in recognition than in recall, and for items involving less specific information than for items involving increased specific information. As was discussed above, it is believed that the strength of the SPT effect depends on task difficulty, rather than solely on type of test or type of item. In the present experiment, it was found that the combination of type of item and type of test determines the effortfulness of the task and, thus, the strength of SPT effect: The more difficult the task is, the stronger the enactment effect should be. The reason why bizzareness did not affect the SPTs in the Mohr et al. study might be that SPT, compared with VT, is a more elaborated task. As was discussed by Nilsson, Nyberg, Kormi-Nouri, and Rönnlund (1995), SPTs are goal-directed tasks, and therefore it is reasonable to assume that the elaboration or the enrichment used for SPTs should be quantitatively or qualitatively different than that used for VTs. It can be reasoned that the conceptual integration used in the present experiment was rich enough to affect SPTs, as it was for VTs. In fact, at recognition, the effect was more pronounced for SPTs than for VTs.

In line with the study by Kormi-Nouri (1995), the lack of difference for type of cue in recognition and recall of SPTs indicates that the two components (action verb and object) of SPTs similarly contribute to the effect of episodic integration and are commonly affected by the semantic integration at encoding. On the other hand, for VT/PI items, there was a differential cue effect: noun cues were better than verb cues. This finding indicates that 
if low semantic integration (PIs) and low episodic integration (VTs) are combined, verb and noun do not reveal similar cuing effects; nouns become more effective cues than verbs.

Recognition failure analysis. For the recognition failure analysis, the data were collapsed across the cue factor. One reason for this was that there was no overall effect of type of cue in recognition or recall. Another reason was that there was a similar pattern of recognition failure data for these two types of cue (verb and noun). The data of greatest interest lie in the predicted and observed values of recognition hits conditionalized on recall (shown in the right part of Table 1). To compute the observed values of recognition success, given cued recall, for each condition, a $2 \times 2$ contingency table (Tulving, 1983; Tulving \& Wiseman, 1975) was arranged. In the $2 \times 2$ contingency data, four possible probabilities were represented: (1) successful recognition and successful recall, (2) successful recognition and failure to recall, (3) successful recall and failure to recognize, and (4) failure to recognize and failure to recall. The observed value of $p$ (Recognition|Recall) for each of the conditions in Table 1 equals the ratio of joint probability of successful recognition and successful recall over the overall probability of successful recall. The predicted values were computed on the basis of the Tulving-Wiseman function.

It can be seen in Table 1, as predicted, that the observed value of recognition given recall deviates below the predicted value for the SPT/WI condition and above the predicted value for the VT/PI condition. In general, however, the differences between the observed and the predicted values are quite small. The significance of these differences was evaluated in two different ways. First, a binomial test (see Neely \& Payne, 1983) revealed that the difference between the observed and the predicted values was significant $(p<.05)$ only in the SPT/WI condition. As expected, the deviation for SPT/WI items was below the function. Thus, the combination of high semantic and episodic integration results in a higher degree of independency between recognition and recall. This is, thus, further support for the notion by Kormi-Nouri (1994, 1995 ) that encoding enactment acts as an episodic integration to increase the linkage between verb and noun. For VT/PI items, the results did not reach significance. But, as expected, the direction of deviation was above the function, reflecting that a low semantic and episodic integration results in a higher dependence between recognition and recall. The binomial test has been extensively used in evaluating differences between observed and predicted values in recognition failure experiments (e.g., Ärlemalm, 1996; Ärlemalm \& Nilsson, 1994; Neely \& Payne, 1983). However, this method of analysis might be questioned on the basis that, first, random errors in the recognition level affect not only the observed value but also the theoretical one. It is therefore inappropriate to treat the situation as one of random variation about a fixed theoretical value. Second, the analysis takes no account of the fact that $p(\mathrm{Rn} \mid \mathrm{Rc})$ is mathematically constrained not to exceed $p(\mathrm{Rn}) / \mathrm{p}(\mathrm{Rc})$ in cases where $p(\mathrm{Rc})$ exceeds $p(\mathbf{R n})$. In order to come to grips with these difficulties with the binomial test, a measure proposed by Nilsson and Gardiner (1993) was also employed in order to evaluate the significance of the differences between the observed and predicted values in this experiment. This measure, called the critical ratio (CR), is expressed as the ratio between single difference scores between the observed and predicted values and the standard deviation for the whole set of difference scores in a large database. When this measure was applied to the four conditions of the present experiment by entering the present difference scores into the Nilsson and Gardiner large database, none of the differences obtained were found to be significant.

The direction of the deviation for overall WIs and PIs was thus in line with the prediction. For WI items, there was a deviation below the function, whereas for PI items, there was a deviation above the function. However, unlike in the study by Svensson and Nilsson (1989), overall SPTs and VTs did not seem to be different with respect to the deviation from the Tulving-Wiseman function. Svensson and Nilsson found a significant deviation below the function for SPTs and above the function for VTs. In the present experiment, there was no deviation for overall SPTs, and there was a very slight and nonsignificant deviation for overall VTs. The possible explanation for this is that the manipulation of semantic integration in the present experiment was responsible for the discrepancy. In the present study, there were two types of integration (WI and PI) for SPTs and VTs. There was no such manipulation in the Svensson and Nilsson study. This becomes more important when, as in the present study, SPTs are affected by level of semantic integration in both recognition and recall, but VTs are affected only in recall. These findings also indicate that it is the integration per se that makes SPT encoding different from VT encoding. In line with this reasoning, there was a slight and nonsignificant deviation in both SPT/PI and VT/WI conditions. This meant that either episodic or semantic integration may result in a moderate dependency between recognition and recall, conforming closely with the Tulving-Wiseman function.

\section{EXPERIMENT 2}

The main purpose of Experiment 2 was to test the idea of interrelation between episodic integration (SPT encoding) and semantic integration (WI encoding) with respect to the exception of cue overlap in the recognition failure paradigm. As was discussed earlier, an increased amount of cue overlap is known to increase the degree of dependence between recognition and recall (Nilsson et al., 1988; Tulving, 1983). As has been demonstrated by Nilsson and Shaps $(1980,1981)$ and Nilsson, Dinniwell, and Tulving (1987), knowledge of the contextual cue at the time of the first test is the basic prerequisite for the occurrence of cue overlap. Svensson and Nilsson (1989) 
used a reversed order of recognition and recall tests (i.e., first, the recall test was given, and second, the recognition test) to meet the criterion for the cue overlap exception. In their experimental condition of cue overlap, they failed to find different patterns for SPTs and VTs with respect to the degree of dependence between recognition and recall: For both SPTs and VTs, there was an increased dependence between recognition and recall in the case of cue overlap.

In the present experiment, a different experimental condition of cue overlap was employed. In relation to the Tulving-Wiseman function, the order of tests remained untouched (i.e., recognition preceded recall). Instead, at the recognition test, both the contextual cue and the copy cue (i.e., the whole item) were presented to subjects, whereas at recall test, the contextual cue was presented to subjects for recall of the target. If the subjects had knowledge of the contextual cue at recognition, when recognition was presented as a first test and not as a second test, it was expected to have a real cue overlap condition, and thereby to have an increased recognition hit rate (in comparison with the no-cue overlap condition in Experiment 1). Furthermore, in this new experimental condition of cue overlap, subjects received the contextual cue at recall for a second time, thereby producing the cue overlap for recall as well as for recognition. It was hypothesized that if there is a strong integration for SPT/WIs (because of the combination of semantic and episodic integration), the independence between recognition and recall would not be affected by using the cue overlap condition. That is, for SPT/WIs, there would still be a deviation below the Tulving-Wiseman function. On the other hand, for VT/PIs, we expected an additional dependence between recognition and recall (i.e., a higher deviation above the Tulving-Wiseman function) in the cue overlap condition, compared with the no-cue overlap condition.

\section{Method}

Design. The design of Experiment 2 was similar to that of Experiment 1 . However, there were some exceptions: First, as was discussed above, the whole item (verb and noun together) was presented at the recognition test. Second, to increase the reliability of data, the number of items (Experiment 1, 48; Experiment 2, 72) and the number of subjects (Experiment 1, 24; Experiment 2, 36) were increased.

Subjects. Thirty-six students were randomly assigned to two recall conditions: verb cue and noun cue. Their age ranges were 20-38 $(M=28.44)$ for the verb cue condition and 20-45 $(M=$ 28.50 ) for the noun cue condition. The subjects were not different with respect to the vocabulary test $(p>.40)$. They participated in the experiment in partial fulfillment of a course requirement.

Materials and Procedure. A new list of 72 commands with two presentation orders comprised TBR items. The items were selected from those used in the normative data (Kormi-Nouri, 1995). Half of the items were selected as WIs and the other half as PIs. Half of the WIs or PIs were encoded as SPTs, and the other half, as VTs. Again, not more than two WIs or PIs, and two SPTs or VTs, followed in a successive order. The procedure for the presentation of the study list and the tests were the same as those in Experiment 1 (viz., in-
Table 2

Mean Recognition (Rn) and Cued Recall (Rc) Probabilities and Observed and Predicted Conditional Recognition Probabilities for Experiment 2

\begin{tabular}{|c|c|c|c|c|c|c|c|}
\hline \multirow[b]{2}{*}{ Condition } & \multicolumn{2}{|c|}{ Rn } & \multicolumn{2}{|c|}{$\mathrm{Rc}$} & \multicolumn{2}{|c|}{$p(\mathrm{Rn} \mid \mathrm{Rc})$} & \multirow[b]{2}{*}{$N$} \\
\hline & $M$ & $S D$ & $M$ & $S D$ & Observed & Predicted & \\
\hline SPT/WI & .92 & .08 & .87 & .08 & $.93 \dagger$ & .96 & 648 \\
\hline $\mathrm{SPT} / \mathrm{PI}$ & .90 & .08 & .65 & .17 & .95 & .95 & 648 \\
\hline VT/WI & .73 & .19 & .73 & .18 & $.80^{*}$ & .83 & 648 \\
\hline $\mathrm{VT} / \mathrm{PI}$ & .64 & .22 & .34 & .19 & $.84^{*}$ & .77 & 648 \\
\hline
\end{tabular}

tentional learning, individual testing of subjects, and rate of item presentation).

\section{Results and Discussion}

As in Experiment 1, a strict scoring of recall was adopted. The results from Experiment 2 are shown in Table 2.

Overall analysis for recognition and recall. As can be seen from this table, the pattern of results, in some respects, is similar to that obtained in Experiment 1. A 2 (list) $\times 2$ (type of item: WI $/ \mathrm{PI}) \times 2$ (type of encoding: SPT/VT) $\times 2$ (type of test: recognition/recall) ANOVA was performed to evaluate the data. The data were collapsed across the type of cue, because there was no difference for this factor in recognition test. Since there was no difference between the two lists $(p>.30)$, the data were also collapsed across this factor. As in Experiment 1, memory for WIs was better than memory for PIs $[F(1,35)=$ $\left.154.81, M S_{\mathrm{e}}=4.81, p<.001\right]$, SPT memory was better than VT memory $\left[F(1,35)=129.89, M S_{\mathrm{e}}=9.66, p<\right.$ $.001]$, and recognition was higher than recall $[F(1,35)=$ $\left.72.07, M S_{\mathrm{e}}=7.37, p<.001\right]$. The effect of semantic integration was more pronounced in recall than in recognition $\left[F(1,35)=105.76, M S_{\mathrm{e}}=3.47, p<.001\right]$. Both SPT and VT recall were affected by semantic integration, although the effect was more pronounced for VTs than for SPTs, and less pronounced than that in Experiment 1. The SPT effect was more pronounced in recognition of WIs, but it was more pronounced in recall of PIs $[F(1,35)=$ $\left.3.60, M S_{\mathrm{e}}=4.07, p=.07\right]$. There were also some discrepancies between the results of two experiments. Unlike in Experiment 1, in Experiment 2, semantic integration did not affect SPTs at recognition. Furthermore, in both cue conditions, there was no difference between recognition and recall with respect to the SPT effect. Also, there was no difference between S PTs and VTs with respect to type of cue (verb or noun).

A separate ANOVA of the cued recall data showed that there was no difference between verb cued recall and noun cued recall for both SPTs and VTs. There was only one marginal interaction of type of cue and type of item $(p=$ $.06)$ : For WIs, verb cues were better than noun cues.

In general, the pattern of data of Experiment 2 was in agreement with that in Experiment 1. For the purpose of the present study, the most important finding was the in- 
terrelation between SPTs and WIs: This was indicated by the finding of the highest performance for SPT/WIs in both recognition and recall. On the other hand, the worst performance was found for VT/PIs. Furthermore, the findings of the larger SPT effect for well-integrated items at recognition, and for poorly integrated items at recall, once again, implies that the effect of enactment is not determined only by type of test. Rather, both type of test and type of item determine the effectiveness of enactment. In general, the SPT effect can be viewed as producing integration between the components of SPTs, but, depending on type of item and type of test, it may act differently. For well-integrated items, recognition requires more episodic integration than recall does, whereas for poorly integrated items, recall requires more episodic integration than recognition does.

The larger SPT effect in recognition than in recall found in the noun cue condition of Experiment 1 was not observed in Experiment 2. In addition, the difference between verb cue and noun cue for VT encoding and not for SPT encoding, found in Experiment 1, was not seen in Experiment 2. Thus, it may be concluded that, in Experiment 1 , the cue difference for SPT and VT encoding was responsible for the finding of a larger SPT effect in recognition than in recall in the noun cue condition: In the noun cue condition, compared with the verb cue condition, recognition of VT/PIs was reduced, whereas recall of VT/PIs was increased.

Recognition failure analysis. As in Experiment 1, since there was no difference between the verb cued recall and noun cued recall conditions, the recognition failure data, in Experiment 2, were collapsed across the cue factor. The observed and predicted values for each condition were computed in the same way as in Experiment 1. In general, for different type of items and different type of encodings, both observed and predicted values increased in comparison with those in Experiment 1. Clearly, this must have been due to the cue overlap condition in both recognition and recall. However, as was predicted, the differences between the observed and predicted values showed different patterns of data with respect to type of item and type of encoding. As in Experiment 1 , the binomial test (Neely \& Payne, 1983) revealed that, in the SPT/WI condition, the observed value was significantly lower than the predicted value (i.e., a deviation below the function; $p<.01)$. This indicates that the combination of high semantic and episodic integration produced a strong integration in such a way that even the cue overlap condition could not affect the amount of independency between recognition and recall. For VT/PI items, the observed value was significantly larger than the predicted value (i.e., a deviation above the function; $p<.05$ ). Although the direction of the effect was the same in both experiments, the effect reached statistical significance in Experiment $2(p<.05)$ but not in Experiment $\mathrm{l}$. As was expected, for VT/PI items, the cue overlap condition resulted in a stronger dependence between recognition and recall. Because of the problems with the binomial test discussed earlier, we also applied the critical ratio measure to the present data by entering the difference scores between observed and predicted values of recognition failure into the Nilsson and Gardiner (1993) large database. This analysis revealed no significant deviation from the Tulving-Wiseman function for any of the four conditions of the present experiment.

There might be two explanations for the findings of significant deviations in Experiment 2: (1) the increase of the number of items and of the number of subjects, and (2) the cue overlap condition. As noted by Nilsson et al. (1988), any dependency between recognition and recall is due to three components: item component, subject component, and item-subject interaction. Thus, any increase of the number of items and/or the number of subjects should result in more reliable data. It can therefore be argued that, because of an increase in number of items and subjects in Experiment 2, the findings were more reliable than those of Experiment 1. The cue overlap condition might be a second reason for the findings of significant deviations, since it produces a case of greater dependency between recognition and recall (Nilsson et al., 1988; Tulving, 1983).

\section{EXPERIMENT 3}

In Experiments 1 and 2, there was a potential problem regarding the position that the recognition-failure function is an appropriate measure of dependency between recognition and recall, because recognition rates differ across SPT and VT encoding. In addition, the comparison of Experiments 1 and 2 could be assessed more properly if cue overlap and no-cue overlap conditions were compared in a single experiment. Experiment 3 was therefore designed to address these issues. To equate the levels of recognition for SPTs and VTs, VTs were recognized and recalled immediately after the presentation of the study list, whereas SPTs were recognized and recalled with a delay. To provide a within-subjects design with respect to the cue variable, half of the complete TBR items were presented at recognition test (i.e., verb and noun together), whereas for the other half of the items, only nouns were presented. Since there was no difference between verb cues and noun cues in the recognition failure data of Experiments 1 and 2, only verb cues (or noun targets) were used in Experiment 3.

\section{Method}

Design. The design of Experiment 3 was 2 (type of cue: cue overlap vs. no-cue overlap) $\times 2$ (type of integration: WI vs. PI) $\times 2$ (type of encoding: SPT vs. VT) $\times 2$ (type of test: recognition vs. recall). All factors were within-subjects variables.

Subjects. Thirty-two university students were used as subjects. Mean age was 25.94 years $(21-41)$. Subjects participated to fulfill a course requirement

Materials. A new list of 88 commands with two presentation orders (with respect to both SPT-VT order and complete-part order) 
comprised the TBR items. The items were selected from those used in the normative data (Kormi-Nouri, 1995). Half of the items were selected as WIs, and the other half, as PIs. Half of the WIs or PIs were encoded as SPTs, and the other half, as VTs. Again, not more than two WIs or PIs, and two SPTs or VTs, followed in a successive order. At recognition test, for each type of encoding integration condition, half of the items were completely presented (cue overlap condition), and the other half were partly presented (no-cue overlap condition).

Procedure. As was mentioned above, to equate the levels of recognition performance for SPTs and VTs, an immediate recognition test was used for VTs, and a delayed recognition test was used for SPTs. To do so, all subjects first received SPTs as TBR items. Following the presentation of SPTs, the vocabulary test (as a distractor task) was conducted. Then, VTs were presented as TBR items. Immediately after the presentation of VTs, the subjects were first given a recognition test, and second, a recall test (verb cue test) for verbal items. Following the recognition and recall tests for VTs, a second distractor task was given to the subjects: to write down as many capital cities of the whole world as possible in any order for $5 \mathrm{~min}$. Finally, the subjects were given first a recognition test and second a recall test for SPTs. It should be noted that the mean interval between the SPT encoding and the recognition test for SPTs was 28 min (22-32). Experiment 3 was similar to Experiments 1 and 2 with respect to intentional learning, individual testing of subjects, and rate of item presentation.

\section{Results and Discussion}

As in Experiments 1 and 2, the scoring of recall was strict. The results from Experiment 3 are shown in Table 3.

Overall analysis for recognition and recall. The main purpose for Experiment 3 was to replicate the results of Experiments 1 and 2 with the levels of recognition equated. A comparison between Experiment 3 and Experiments 1 and 2 shows that, whereas recognition performance of VTs increased, recognition of SPTs decreased. Thus, as a consequence of using an immediate recognition test for VTs and a delayed recognition test for SPTs, the levels of recognition for SPTs and VTs were almost successfully equalized. As can be seen from the table, the pattern of results (the hit rate data) are similar to those obtained in Experiments 1 and 2. The only noticeable difference between Experiment 3 and Experiments 1 and 2 is the mag- nitude of SPT effect at recognition test: Regardless of the cue conditions in Experiment 3, at recognition, the SPT effect was smaller than in Experiments 1 and 2. However, it is noteworthy that there is a superiority for SPTs over VTs, at least for WI items, despite the fact that SPTs were tested after a delay whereas VTs were tested immediately. It should also be noted that the comparison of Experiment 3 with Experiments 1 and 2 showed the SPT effect to have decreased considerably in recognition, but not in recall. This could explain the finding of larger SPT effect in recall than in recognition.

A 2 (list) $\times 2$ (type of cue: cue overlap/no-cue overlap) $\times 2$ (type of encoding: SPT/VT) $\times 2$ (type of integration: WI/PI) $\times 2$ (type of test: recognition/recall) ANOVA was performed in order to evaluate the data of Experiment 3 . The data were collapsed across the list factor, since there was no difference between the two lists $(p>.70)$ and there were no interactions involving this factor. The ANOVA summary is presented in Table 4.

With regard to the cue conditions, memory performance, in both recognition and recall, was better in the cue overlap condition than in the no-cue overlap condition. As was expected, the finding of better recognition (both with hit rates and with hit rates - false alarms) in the cue overlap condition than in the no-cue overlap condition indicates that the subjects, in the cue overlap condition, had knowledge of the contexual cues at recognition, and we therefore were dealing with a real cue overlap condition. In the no-cue overlap condition, the SPT effect was not different for WIs and PIs, whereas in the cue overlap condition, it was larger for PIs than for WIs (the three-way interaction of cue $X$ encoding $\times$ item). Another interpretation of this interaction is that, in both cue conditions, the semantic integration effect (the difference between WI and PI) was the same for VTs, whereas for SPTs, it was larger in the no-cue overlap condition than in the cue overlap condition. In both cue conditions, at recognition, there was a small semantic integration effect, whereas at recall, there was a large semantic integration effect (the two-way interaction of integration $x$

Table 3

Mean Recognition (Rn) and Cued Recall (Rc) Probabilities and Observed and Predicted Conditional Recognition Probabilities for Experiment 3

\begin{tabular}{|c|c|c|c|c|c|c|c|c|c|}
\hline \multirow[b]{3}{*}{ Condition } & \multicolumn{4}{|c|}{ Rn } & \multirow{2}{*}{\multicolumn{2}{|c|}{$\mathbf{R c}$}} & \multirow{2}{*}{\multicolumn{2}{|c|}{$p(\mathbf{R n} \mid \mathbf{R} \mathbf{c})$}} & \multirow[b]{3}{*}{$N$} \\
\hline & \multicolumn{2}{|c|}{ Hit rate } & \multicolumn{2}{|c|}{ False alarm } & & & & & \\
\hline & $M$ & $S D$ & $M$ & $S D$ & $M$ & $S D$ & Observed & Predicted & \\
\hline \multicolumn{10}{|c|}{ No-Cue Overlap } \\
\hline SPT/WI & .83 & .13 & .04 & .06 & .90 & .12 & $.85 \dagger$ & .90 & 352 \\
\hline $\mathrm{SPT} / \mathrm{PI}$ & .73 & .19 & .07 & .09 & .53 & .23 & .83 & .82 & 352 \\
\hline VT/WI & .73 & .18 & .07 & .10 & .70 & .18 & .82 & .82 & 352 \\
\hline $\mathrm{VT} / \mathrm{PI}$ & .74 & .15 & .11 & .11 & .27 & .21 & .90 & .84 & 352 \\
\hline \multicolumn{10}{|l|}{ Cue Overlap } \\
\hline SPT/WI & .89 & .09 & .12 & .09 & .90 & .14 & $.90^{*}$ & .94 & 352 \\
\hline SPT/PI & .85 & .15 & .05 & .11 & .69 & .24 & .91 & .90 & 352 \\
\hline $\mathrm{VT} / \mathrm{WI}$ & .84 & .12 & .09 & .09 & .78 & .15 & .90 & .91 & 352 \\
\hline VT/PI & .83 & .14 & .07 & .11 & .38 & .22 & $.95 \dagger$ & .90 & 352 \\
\hline
\end{tabular}


Table 4 Summary ANOVA for Data of Experiment 3

\begin{tabular}{lccrc}
\multicolumn{1}{c}{ Source } & $d f$ & $M S_{\mathrm{e}}$ & \multicolumn{1}{c}{$F$} & $P$ \\
\hline Cue & 1,31 & 1.52 & 84.20 & $<.001$ \\
Encoding & 1,31 & 4.16 & 65.30 & $<.001$ \\
Integration & 1,31 & 2.58 & 224.33 & $<.001$ \\
Test & 1,31 & 5.72 & 69.13 & $<.001$ \\
Encoding $\times$ test & 1,31 & 2.69 & 48.34 & $<.001$ \\
Integration $\times$ test & 1,31 & 1.60 & 242.73 & $<.001$ \\
Integration $\times$ cue & 1,31 & 1.22 & 10.23 & $<.01$ \\
Integration $\times$ cue $\times$ test & 1,31 & 1.03 & 5.53 & $<.05$ \\
Encoding $\times$ integration $\times$ test & 1,31 & 2.67 & 17.38 & $<.001$ \\
Encoding $\times$ integration $\times$ cue & 1,31 & 1.92 & 5.87 & $<.05$ \\
\hline
\end{tabular}

test). This effect was more pronounced in the no-cue overlap condition than in the cue overlap condition (the three-way interaction of cue $X$ integration $\times$ test). At recognition, the SPT effect was larger for WIs than for PIs, whereas at recall, it was larger for for PIs than for WIs (the three-way interaction of encoding $X$ integration $X$ test). From these interactions, it may be concluded that the SPT effect and the semantic integration effect become more efficient when the tasks are more effortful (e.g., no cue overlap vs. cue overlap, recall vs. recognition, or PI vs. WI).

Two separate ANOVAs were also conducted for each of the cue conditions. These $2 \times 2 \times 2$ ANOVAs revealed that the main effects and the interactions were significant in both cue conditions. The only difference between the two cue conditions was related to the interaction between type of encoding and type of item: In the cue overlap condition, the SPT effect was larger for PIs than for WIs. Also, the semantic integration effect was larger for VTs than for SPTs. These effects were not significant in the no-cue overlap condition.

It should be noted that the recognition data of Experiment 3 were corrected for false alarms (i.e., hit rates false alarms) and were again compared with the recall data. A $2 \times 2 \times 2 \times 2$ ANOVA conducted on these data showed similar findings to those found for recognition with hit rates. ${ }^{2}$

Recognition failure analysis. The observed and predicted values computed for each condition was similar to those in Experiments 1 and 2. Clearly, the results are in agreement with those of Experiments 1 and 2 with respect to the direction of the deviations from the TulvingWiseman function. The binomial test revealed that in both cue conditions, for SPT/WIs, the observed value was significantly lower than the predicted value: a deviation below the function (in the no-cue overlap condition, $p<.01$; in the cue overlap condition, $p<.05$ ). Once again, this indicates that the combination of high semantic and episodic integration produces strong integration and thereby independence between recognition and recall. For VT/PI items, in both cue conditions, the observed value was larger than the predicted value: a deviation above the function. It should be noted that this difference reached significance only in the cue overlap condition $(p<.05)$, indicating that there would be an additional dependence between recognition and recall in the cue overlap condition. Again the difference scores obtained were also entered into the Nilsson and Gardiner (1993) database and the CR measure was employed. It was again demonstrated that the none of the difference scores reached significance.

Using the Nilsson and Gardiner (1993) database as a means to evaluate the differences obtained here makes the CR measure a very conservative test. A new database was therefore created by including the data obtained in the 16 conditions of the three experiments reported here and data from the critical 2 conditions reported by Svensson and Nilsson (1989). Although not at all as large as the Nilsson and Gardiner database, the database obtained this way consists of 7,208 individual observations. The rationale for including only these data in the database was that these are the only studies reported in which enactment/nonenactment conditions have been used in recognition failure experiments. Employing the critical ratio measure on this database revealed significant differences above the Tulving-Wiseman function for the VT/PI condition in Experiment 2.

The findings of deviation above the function for VT/PIs indicate that the combination of low semantic and episodic integration produces a poorer integration and thereby dependence between recognition and recall. Thus, although the levels of recognition performance for SPTs and VTs were almost equalized in Experiment 3, the findings of larger independence for SPT/WIs and larger dependence for VT/PIs were again replicated in both cue conditions. It should be noted that, as in Experiments 1 and 2, a moderate dependence was found for SPT/PIs and VT/WIs in botit cue conditions.

\section{GENERAL DISCUSSION}

The present experiments yielded two main results. First, a robust relationship between encoding enactment and semantic integration was observed. This means that these two types of processing show similar effects and might be regarded as two ways of affecting the same underlying memory processing. Second, a new systematic and analytic way of varying the degree of integration for recognition failure studies was developed.

The findings of high independence between recognition and recall of well-integrated items following encoding enactment indicate that SPT encoding may involve an integrative function. That is, when subjects perform a learning task, both action and object are integrated as one entity. The action (motor component: verb) refers to the subject, and the object refers to the environment. Thus, a two-way interaction between the subject and the environment, an active learning, occurs at encoding enactment. From an information processing view, SPT encoding produces a memory trace for both verb and noun. For each individual SPT item, verb and noun are encoded in 
a single memory unit or in strongly connected units. This interaction between the subject and the environment or integration between verb and noun has been proposed as episodic integration (Kormi-Nouri, 1995). On the other hand, when there is no such encoding enactment (VT encoding), a passive learning occurs. Learning materials (environment) are presented to the subject, but the subject does not manipulate them. Thus, there is only a oneway interaction: The environment affects the subject, but the subject does not affect the environment. From an information processing perspective, for each VT item, verb and noun are encoded in separately or loosely connected memory units. Support for this idea in the present study was the observation of the highest dependence (or the lowest independence) between recognition and recall of poorly integrated items following VT encoding.

An interesting finding is that even in the cue overlap conditions (compared to the no-cue overlap conditions) the same pattern of data was observed. As was noted in the introduction, a cue overlap condition increases the degree of dependency between recognition and recall (Nilsson et al., 1988; Tulving, 1983). However, in the present study, the cue overlap conditions, in both between- and withinsubjects designs, did not affect the independence between recognition and recall of SPT/WIs. This indicates that there is a robust integration between these two types of items (i.e., SPTs and WIs) in such a way that incrementing the amount of dependence between recognition and recall by creating a cue overlap condition did not change the pattern of data for these items. Furthermore, as was expected, an additional dependence between recognition and recall of VT/PI items was caused by the cue overlap condition, in comparison with the no-cue overlap condition.

In the present experiments as well as in the previous experiments (Kormi-Nouri, 1995), the stable finding of an interaction of type of encoding, type of item, and type of test revealed that the enactment effect is determined by two factors: type of item and type of test. Whereas Svensson and Nilsson (1989) and Nilsson and Craik (1990) found that the enactment effect was higher in recall than in recognition, Mohr et al. (1989) found that the enactment effect was higher in recognition than in recall. The possible explanation for this discrepancy may involve the type of items used in these studies. It should be noted that since the lists of items in the studies mentioned were not available, the explanation proposed here is based on the data of the present experiments: The consistent finding was that, for well-integrated items, the effect of enactment is larger in recognition than in recall, whereas, for poorly integrated items, the enactment effect is larger in recall than in recognition. For well-integrated items, recognition is more difficult than recall, whereas, for poorly integrated items, recall is more difficult than recognition. Thus, the effect of enactment is due to the nature of task: The more difficult the task, the more the enactment that is required. The function of encoding enactment is to integrate verb and noun episodically, thereby producing specific information.
The relationship between semantic and episodic integration may allow us to vary analytically the degree of integration in the recognition failure experiments. In only one study (Ärlemalm \& Nilsson, 1994) was there an attempt to obtain a more direct index of the degree of integration. Ärlemalm and Nilsson manipulated the degree of integration by type of encoding instruction (shallow or deep) and type of recall confidence (high or low). Ärlemalm and Nilsson demonstrated that a very strong integration (i.e., deep encoding and high recall confidence) produces a deviation below the function, whereas a very poor integration (i.e., shallow encoding and low recall confidence) produces a deviation above the function.

In the present study, we manipulated the degree of integration only at the study phase. The combination of SPTs and WIs produced the highest degree of integration, whereas the combination of VTs and PIs produced the lowest degree of integration. Either the combination of SPTs and PIs or VTs and WIs produced a moderate integration. In line with the findings of Ärlemalm and Nilsson (1994), this variation of the degree of integration may allow us to find both positive and negative significant deviations. Of 302 conditions reported by Nilsson and Gardiner (1993), 275 conditions fit the TulvingWiseman function, 25 conditions deviated above the function, and 2 conditions deviated below the function. Nilsson and Gardiner concluded that exceptions to the recognition function can be defined as positive deviations. They considered the two negative deviations as reflecting some unknown factor or experimental error. In the present study, 7 significant negative deviations and 3 significant positive deviations were found. This implies that the manipulation of both semantic and episodic integration at encoding can reveal both extreme cases of deviations. Encoding of VT/PI items, in which the contextual cues are poorly related to their target words, leads to a positive deviation. Encoding of SPT/WI items, in which the contextual cues are highly related to their target words, leads to a negative deviation.

Thus, if the degree of integration between contextual cues and their targets at encoding is systematically increased, a negative deviation is found. It might be argued that the negative deviations for SPT/WI items were due to the ceiling effects found for recognition of these items. However, the findings of negative deviations for VT/WI and overall WI items, in which there were no ceiling effects at recognition, refute the argument of experimental error. Clearly, this idea should be verified by further experimental scrutiny.

\section{REFERENCES}

ÄrLEMALM, T. (1996). Recognition failure: The influence of semantic cue-target integration: A short note. European Journal of Cognitive Psychology, 8, 205-214.

Ärlemalm, T., \& Nilsson, L.-G. (1994). Recognition failure and integration. Scandinavian Journal of Psychology, 35, 271-280.

BäCKMAN, L., \& Nilsson, L.-G. (1984). Aging effects in free recall: An exception to the rule. Human Learning, 3, 53-69.

Bäckman, L., \& Nilsson, L.-G. (1985). Prerequisites for lack of age 
difference in memory performance. Experimental Aging Research, 11, 67-73.

BäCKMAN, L., Nilsson, L.-G., \& Chalom, D. (1986). New evidence on the nature of the encoding of action events. Memory \& Cognition, 14, 339-346.

Bäckman, L., Nilsson, L.-G., Herlitz, A., Nyberg, L., \& StigsdotTER, A. (1991). A dual conception of the encoding of action events. Scandinavian Journal of Psychology, 32, 289-299.

BäCKMan, L., Nilsson, L.-G., \& Kormi-Nouri, R. (1993). Attentional demands and recall of verbal and color information in action events. Scandinavian Journal of Psychology, 34, 246-254.

COHEN, R. L. (1981). On the generality of some memory laws. Scandinavian Journal of Psychology, 22, 267-282.

CoHEN, R. L. (1983). The effect of encoding variables on the free recall of words and action events. Memory \& Cognition, 11, 575-582.

COHEN, R. L. (1989). Memory for action events: The power of enactment. Educational Psychology Review, 1, 57-80.

COHEN. R., L., \& STEWART, M. (1982). How to avoid developmental effects in free recall. Scandinavian Journal of Psychology, 23, 9-16.

ENGELKAMP, J., \& KRUMNACKER, H. (1980). Imaginale and motorische Prozesse beim Behalten verbalen Materials [Image and motor processes in memory for verbal materials]. Zeitschrift für experimentelle und angewandte Psychologie, 27, 511-533.

ENGElKamP, J., \& Zimmer, H. D. (1983). Zum Einfluß von Wahrnehmen und Tun auf das Behalten von Verb-Objekt-Phrasen [On the influence of perception and action on memory for verb-object phrases]. Sprache \& Kognition, 2, 117-127.

ENGELKAMP, J., \& ZIMMER, H. D. (1984). Motor program information as a separate memory unit. Psychological Research, 46, 283-299.

HELSTRUP, T. (1986). Separate memory laws for recall of performed acts? Scandinavian Journal of Psychology, 27, 1-29.

HelstruP, T. (1987). One, two, or three memaries? A problem solving approach to memory for performed acts. Acta Psychologica, 66, 3768.

KoRMI-NourI, R. (1994). Memory for action events: An episodic integration view. Unpublished doctoral dissertation, Umeå University, Sweden.

KoRMI-NOURI, R. (1995). The nature of memory for action events: An episodic integration view. European Journal of Cognitive Psychology, 7, 337-363.

Kormi-Nouri, R., Nilsson, L.-G., \& Bäckman, L. (1994). The dual conception view reexamined: Attentional demands and the encoding of verbal and physical information in action events. Psychological Research, 57, 42-46.

Kormi-Nouri, R., NYberG, L., \& Nilsson, L.-G. (1994). The effect of retrieval enactment on recall of subject-performed tasks and verbal tasks. Memory \& Cognition, 22, 723-728.

Mohr, G., EngelKamP, J., \& Zimmer, H. D. (1989). Recall and recognition of self-performed acts. Psychological Research, 51, 181-187.

NeELY, J. H., \& PAYNE, D. G. (1983). A direct comparison of recognition failure rates for recallable names in episodic and semantic memory tests. Memory \& Cognition, 11, 161-171.

NiLsson, L.-G., \& CRAIK, F. I. M. (1990). Additive and interactive effects in memory for subject-performed tasks. European Journal of Cognitive Psychology, 2, 305-324.
Nilsson, L.-G., Dinniwell, M., \& Tulving, E. (1987). Recognition failure of categorized words. Memory \& Cognition, 15, 389-396.

NiLSSON, L.-G., \& GARDINER, J. M. (1993). Identifying exceptions in a database of recognition failure studies from 1973 to 1992 . Memory \& Cognition, 21, 397-410.

Nilsson, L.-G., Law, J., \& Tulving, E. (1988). Recognition failure of recallable unique names: Evidence for an empirical law of learning and memory. Journal of Experimental Psychology: Learning, Memory, \& Cognition, 14, 266-277.

Nilsson, L.-G., NyberG, L., Kormi-Nouri, R., \& Rönnlund, M. (1995). Dissociative effects of elaboration on memory of enacted and non-enacted events: A case of negative effect. Scandinavian Journal of Psychology, 36, 225-231.

NiLSSON, L.-G., \& SHAPS, L. P. (1980). A functional view of memory. In F. Klix \& J. Hoffmann (Eds.), Cognition and memory: Interdisciplinary research of human memory activities (pp. 40-46). Berlin: Deutscher Verlag der Wissenschaften.

NiLSSON, L.-G., \& SHAPS, L. P. (1981). A reconstructive processing interpretation of the recognition failure phenomenon. Acta Psychologica, 47, 25-37.

SALTZ, E., \& DONnENwerTh-Nolan, S. (1981). Does motoric imagery facilitate memory for sentences? A selective interference test. Journal of Verbal Learning \& Verbal Behavior, 20, 322-332.

SHAPS, L. P., \& NiLSSON, L.-G. (1980). Encoding and retrieval operation in relation to age. Developmental Psychology, 16, 636-643.

SVENSSON, T., \& NiLsSON, L.-G. (1989). The relation between recognition and cued recall in memory of enacted and nonenacted information. Psychological Research, 51, 194-200.

Tulving, E. (1983). Elements of episodic memory. New York: Oxford University Press.

Tulving, E., \& Thomson, D. M. (1973). Encoding specificity and retrieval processes in episodic memory. Psychological Review, 80, 352-373.

Tulving, E., \& Wiseman, S. (1975). Relation between recognition and recognition failure of recallable words. Bulletin of the Psychonomic Society, 6, 79-82.

\section{NOTES}

1. Tulving and Wiseman (1975) examined the results of a number of experiments and described the resı!ts with the function $P(\mathrm{Rn} \mid \mathrm{Rc})=$ $p(\mathbf{R n})+c\left[p(\mathbf{R n})-p(\mathbf{R n})^{2}\right]$, where $P(\mathbf{R n} \mid \mathbf{R c})$ stands for the probability of recognition conditionalized on recall, and $p(\mathrm{Rn})$ stands for recognition hit rate. The constant $c$ is equal to .5 , which was calculated by the method of least squares for the data available to Tulving and Wiseman and confirmed by Nilsson and Gardiner (1993) in a database of recognition failure studies from 1973 to 1992.

2. This correction could not be properly done in Experiments 1 and 2 , since SPTs and VTs were tested together and distractors could not be separately related to each of these tasks. The overall false alarms were similar in all three experiments (Experiment 1, .08; Experiment 2, .06; Experiment 3, .08).

(Manuscript received May 2, 1996; revision accepted for publication March 21, 1997.) 